\title{
DEMO divertor limitations during and in between ELMs
}

\author{
RP Wenninger ${ }^{1,2}$, M Bernert ${ }^{1}$, T Eich $^{1}$, E Fable $^{1}$, G Federici $^{2}$, \\ A Kallenbach ${ }^{1}$, A Loarte ${ }^{3}$, C Lowry ${ }^{2}$, D McDonald ${ }^{2,4}, \mathbf{R} \mathbf{N e u}^{1,2}$, \\ T Pütterich ${ }^{1}$, P Schneider ${ }^{1}$, B Sieglin ${ }^{1}$, G Strohmayer ${ }^{1}$, F \\ Reimold $^{1}, \mathrm{M}$ Wischmeier ${ }^{1}$ \\ ${ }^{1}$ Max-Planck-Institut für Plasmaphysik, EURATOM Association, Garching, \\ Germany \\ ${ }^{2}$ EFDA, Garching, Germany \\ ${ }^{3}$ ITER Organization, St. Paul Lez Durance, France \\ ${ }^{4}$ CCFE Culham, EURATOM Association, Culham, United Kingdom \\ E-mail: ronald.wenninger@ipp.mpg.de
}

\begin{abstract}
Operation of DEMO in comparison to ITER will be significantly more demanding, as various additional limitations of physical and technical nature have to be respected. In particular a set of extremely restrictive boundary conditions on divertor operation during and in between ELMs will have to be respected. It is of high importance to describe these limitations in order to consider them as early as possible in the ongoing development of the DEMO concept design. This paper extrapolates the existing physics basis on power and particle exhaust to DEMO.

In phases between ELMs or with mitigated ELMs surface overheating and W sputtering pose challenging boundary conditions. For attached divertor conditions at $90 \%$ total radiation fraction a peak power density of about $15 \mathrm{MW} / \mathrm{m}^{2}$ convected or radiated to the outer divertor is estimated. As this clearly exceeds the tolerable limit, some degree of divertor detachment is regarded as essential for the operation of DEMO. A loss of detachment with a peak power density of more than $30 \mathrm{MW} / \mathrm{m}^{2}$ can not be tolerated for more than a second before the divertor would suffer from a destructive event. The combination of the limitations on the peak power flux density and $\mathrm{W}$ sputtering rate necessitates divertor temperatures less than $4 \mathrm{eV}$.

For uncontrolled ELMs sizes in the order of 100MJ are estimated. Results on ELM broadening from JET suggest that in DEMO an energy density limit of $0.5 \mathrm{MJ} / \mathrm{m}^{2}$ per ELM is exceeded by a factor of about 8 for a large range of relative ELM sizes. This highlights the necessity of a reactor-relevant ELM control technique for DEMO, which is capable of reducing the maximum size of the energy loss per ELM to the divertor by more than an order of magnitude without a strong reduction of confinement.
\end{abstract}

\section{Introduction}

The design and construction of a Demonstration Fusion Power Reactor (DEMO) as a single step between ITER and a fusion power plant is among the most important 
objectives of the EU fusion road map [1]. Limitations associated with the exhaust of power and particles are regarded as one of the ultimate challenges for the design of DEMO. Hence these boundary conditions need to be integrated already into the conceptual design of DEMO, which is currently under development, in favor of a fast track to fusion energy.

Currently the physics of a conventional tokamak divertor in H-mode is not sufficiently understood to have the capability to fully simulate the DEMO divertor. This is the case in particular for the operation with a detached divertor. However it is necessary to specify divertor related boundary conditions in the DEMO conceptual design process as soon as possible. This paper extrapolates the existing physics basis on power and particle exhaust to DEMO. A conventional divertor with W plasma facing components is assumed. As technological basis for armor and heat removal the watercooled monoblock concept [2] has been chosen, which consists of a $\mathrm{W}$ block enclosing a $\mathrm{Cu}$ pipe containing the coolant (section 3.2).

For periods between ELMs or in scenarios with mitigated ELMs the main limited quantities are (i) peak power flux density (ii) divertor $\mathrm{W}$ sputtering rate (iii) core plasma impurity concentration and (iv) displacements per atom due to neutrons. Also at high densities limitations related to radiative instabilities have been observed [3]. Additionally during ELMs there are limitations in similar areas, some of which are more restrictive compared to their inter-ELM equivalents. Only a subset of all limitations is discussed in this publication.

\section{Scenario assumptions}

\subsection{The design option DEMO1}

The reference for this analysis is the European design option DEMO1, which is a near-term, modest power density, pulsed device with a net electric output of 500MW [4]. DEMO1 incorporates a conventional H-mode scenario based on the expected ITER performance with moderate improvements in terms of scenario and employed technologies. Table 1 lists key parameters of the DEMO1 design version from 2013 in comparison to the ITER $\mathrm{Q}=10$ scenario. It is important to mention that this DEMO1 design is in conflict with some divertor limitations described below.

\subsection{The DEMO1 pedestal}

The height of the density and temperature pedestal is very important for a number of divertor limitations. It also determines significantly the fusion performance. However, the extrapolation of these parameters to DEMO is a particular challenge.

There is a number of indications that the Greenwald density limit is related to

processes in the plasma edge [5]. Therefore, it seems natural to relate the pedestal top density to the Greenwald density. It has been assumed that the pedestal top density is $85 \%$ of the Greenwald density. Similar ratios of the pedestal top density to the 
Table 1. Overview of key parameters of the ITER $(\mathrm{Q}=10)$ and DEMO1 reference designs (2013)

\begin{tabular}{l|c|c} 
& ITER $(\mathrm{Q}=10)$ & DEMO1 \\
\hline $\mathrm{R}[\mathrm{m}]$ & 6.2 & 9.0 \\
$\mathrm{~A}$ & 3.1 & 3.6 \\
$\kappa_{x}$ & 1.85 & 1.75 \\
$\delta_{x}$ & 0.48 & 0.50 \\
$B_{T}[\mathrm{~T}]$ & 5.3 & 6.5 \\
$I_{P}[\mathrm{MA}]$ & 15.0 & 16.8 \\
$P_{\text {fus }}[\mathrm{MW}]$ & 500 & 1790 \\
$P_{\text {heat }, \text { add }}[\mathrm{MW}]$ & 50 & 50 \\
$<n_{e}>\left[10^{19} \mathrm{~m}^{-3}\right]$ & 10.1 & 9.3 \\
$n_{\text {GW }}\left[10^{19} \mathrm{~m}^{-3}\right]$ & 11.9 & 8.6 \\
$\beta_{N, \text { tot }}$ & 1.8 & 2.5 \\
Pulse length $[\mathrm{h}]$ & 0.1 & 1.7 \\
$q_{\text {neutron,wall }}\left[M W / m^{2} \mid\right.$ & $\sim 0.5$ & 1.1 \\
Total dpa & $\sim 3$ & $20+30$
\end{tabular}

Greenwald density have been demonstrated in JET [6, 7]. In ASDEX Upgrade even higher ratios have been observed. It is not clear, if a scenario with $n_{\text {ped }} / n_{G W}=85 \%$ can also be realized in DEMO in terms of fueling and edge stability. Applying this ratio to DEMO1, an absolute pedestal top density of $7.3 \times 10^{19} \mathrm{~m}^{-3}$ is obtained. Due to the smaller minor radius at similar plasma current, ITER in the $\mathrm{Q}=10$ scenario is expected to have a higher Greenwald density limit and a pedestal top density of $8 \times 10^{19} \mathrm{~m}^{-3}$. It has to be noted that a degradation of the pedestal pressure at densities close to the density limit as observed in ASDEX Upgrade [8] has not been considered.

In order to extrapolate the DEMO pedestal top temperature a scaling of the pedestal top pressure [9] on the basis of data from H-mode discharges in ASDEX Upgrade, DIII-D and JET has been used. This implies a very large extrapolation in particular in terms of total heating power. Therefore the results are considered to have a large uncertainty. The resulting pedestal top electron temperature for DEMO1 is $8 \mathrm{keV}$. It remains to be examined, if such a high pedestal top pressure is compatible with a pedestal structure, which is stable against MHD instabilities.

\section{Divertor limitations in phases without ELMs}

\subsection{Peak power flux density for attached divertor configuration}

During the last years the characterization of the scrape-off layer (SOL) and divertor heat transport has been significantly developed. Employing infrared thermography the power flux density loading an attached divertor can be measured with high time resolution. The radial divertor power flux density profile fits remarkably well a convolution of an 
exponential and a Gaussian [10]. It is assumed that the exponential function specified by the SOL fall off length at the outer midplane $\lambda_{q}$ mainly represents the processes in the SOL, while the Gaussian specified by its width $S$ (divertor spreading) mainly reflects the processes in the divertor. This assumption is supported by experiments in ASDEX Upgrade [11] revealing for similar discharge conditions similar upstream $\lambda_{q}$ but different values for $S$ resulting from the significant changes in the divertor geometries (Div-I and Div-IIb) and corresponding divertor plasma. It has been shown that for attached divertor configurations the two dimensions $\lambda_{q}$ and $S$ can be combined into the integral width [12]:

$$
\lambda_{\text {int }}:=\frac{\int\left(q(\bar{s})-q_{B G}\right) d \bar{s}}{q_{\max }}=\lambda_{q}+1.64 \times S
$$

$\lambda_{\text {int }}$ is the quantity required to evaluate the peak power flux density.

The separation of physical mechanisms motivates a separate extrapolation of $\lambda_{q}$ and $S$ to DEMO1. Based on IR data from H-mode discharges in JET, DIII-D, ASDEX Upgrade and Alcator C-Mod a scaling of the SOL fall off length has been developed [13]:

$$
\lambda_{q}=0.7 B_{\phi}^{-0.77} q_{95}^{1.05} P_{s e p}^{0.09} R^{0} .
$$

$B_{\phi}$ is the toroidal magnetic field, $q_{95}$ is the safety factor at the surface of $95 \%$ poloidal magnetic flux, $P_{\text {sep }}$ is the power transported across the separatrix by electrons and ions and $R$ is the major radius. For DEMO1 this leads to $\lambda_{q}=0.8 \mathrm{~mm}$. However it has to be noted that $P_{\text {sep }}$ is for DEMO1 more than an order of magnitude higher than the highest values used for the scaling.

An extrapolation of the divertor spreading has to stay speculative, due to the lack of appropriate scalings. Typical values of $\mathrm{S}$ in an open divertor configuration are $0.5 \mathrm{~mm}$ for ASDEX Upgrade (outer divertor Div-I) and 1mm for JET when the outer divertor leg is positioned on a horizontal target plate [14]. We speculate that S scales linear with $\mathrm{R}$. This is backed up by the consideration, that under the assumption of pure diffusive transport in the divertor $\mathrm{S}$ can be written as $l_{x} \sqrt{\chi_{\perp} / \chi_{\|}}$, where $l_{x}$ is the connection length between x-point and target and $\chi_{\perp}$ and $\chi_{\|}$are the perpendicular and parallel heat diffusivities. Further it is stressed that for the closed divertor Div-IIb in ASDEX Upgrade in vertical target operation values for $\mathrm{S}$ of $1.5 \mathrm{~mm}$ are found [14], hence 3 times as large as for the open divertor Div-I. Also it is observed in ASDEX Upgrade L-Mode that at the outer divertor $\mathrm{S}$ is proportional to $1 / B_{\text {pol }}$ [15], which would lead to a reduction of $S_{D E M O 1}$ by a factor of $2\left(B_{\text {pol,DEMO }} \approx 1 \mathrm{~T}, B_{\text {pol,JET }} \approx 0.5 \mathrm{~T}\right)$. All this information may be combined into a speculative prediction for $\mathrm{S}$ in a closed baffled DEMO divertor as

$$
S_{D E M O 1}=S_{J E T, \text { open }} \frac{R_{D E M O 1}}{R_{J E T}} \frac{S_{A U G, \text { closed }}}{S_{A U G, \text { open }}} \frac{B_{\text {pol }, J E T}}{B_{\text {pol,DEMO }}} \approx 4.5 \mathrm{~mm} .
$$

Consequently in the following a power spreading in DEMO1 of $4.5 \mathrm{~mm}$ is used, which in combination with $\lambda_{q}=0.8 \mathrm{~mm}$ leads to an integral width of $\lambda_{\text {int }}=0.8 \mathrm{~mm}+$ $1.64 \times 4.5 \mathrm{~mm} \approx 8 \mathrm{~mm}$ for DEMO1. Taking into account the significant uncertainties 
in the derivation of this number it is obvious that further experimental and theoretical action is needed to improve this situation.

An exact formulation has been proposed for the conversion of $\lambda_{\text {int }}$ into the wetted area $A_{\text {out }}$ on the outer target, defined by $q_{\text {max }, \text { out }}=P_{\text {targ }} / A_{\text {out }}[16]$ :

$$
A_{\text {out }}=\frac{2 \pi R_{S} \lambda_{\text {int }} B_{\theta, m}}{B_{\phi, m}} \frac{\cos \theta_{\perp}}{\sin \left(\alpha+\theta_{\perp}\right)}
$$

Here $R_{S}$ is the major radius at the strike point, which is approximated by $R$ lacking a converged divertor geometry. $B_{\theta, m}$ and $B_{\phi, m}$ are the magnetic field components at the outer midplane separatrix position. The ratio $B_{\theta, m} / B_{\phi, m}$ can be approximated within a few percent by $3 a /\left(R q_{95}\right)$. The geometrical situation at the divertor target is described by the two angles $\alpha$ and $\theta_{\perp}$. $\alpha$ is the inclination of the divertor tiles with respect to the toroidal direction, which is necessary in order to shield leading edges arising due to toroidal gaps between tiles. $\theta_{\perp}$ is the angle between the field line and the toroidal direction in the projection to the plane defined by the toroidal direction and the direction perpendicular to the tile. From an engineers perspective challenging but achievable values for the two angles are $\alpha=1^{\circ}$ and $\theta_{\perp}=3^{\circ}$. The formulation takes into account the angle between the poloidal field and the target, the toroidal wetted fraction $(\approx 75 \%)$ and the width of the SOL at the target. The outer wetted area for DEMO1 calculates to $1.4 m^{2}$.

Assuming a distribution of power between outer and inner target of 2:1 [17], a power spreading in DEMO1 of $4.5 \mathrm{~mm}$ and the complete absence of any radiation results in a peak power flux density for DEMO1 of $150 \mathrm{MW} / \mathrm{m}^{2}$. For DEMO1 with water cooling no experimental data on the power flux density limit under relevant conditions are available. Simulations not accounting for irradiation damage suggest that up to $10 \mathrm{MW} / \mathrm{m}^{2}$ could be tolerated. For the analysis presented here, as a conservative assumption a power flux density limit at the divertor target of $5 \mathrm{MW} / \mathrm{m}^{2}$ is used. Hence, it is extremely desirable to maximize the radiation inside and outside the separatrix. To limit the power flux density at the outer divertor target to $5 \mathrm{MW} / \mathrm{m}^{2}$, a total radiation fraction of $96.5 \%$ would be necessary. In ASDEX Upgrade a total radiation fraction of $85 \pm 10 \%$ has been reached with double radiation feedback control [18]. In the following for DEMO1 a total radiation fraction of $90 \%$ is assumed. It should be noted, that it is not clear, if this is achievable in terms of physics and engineering. Based on such a high radiation level and a power spreading in DEMO1 of $4.5 \mathrm{~mm}$ a peak power flux density of $15 \mathrm{MW} / \mathrm{m}^{2}$ is calculated, which is still significantly above the material limit. As $\mathrm{S}$ is the quantity with the highest uncertainty in this analysis table 2 displays the divertor spreading $S_{\text {requ }}$ to allow for an outer peak heat flux density of $5 \mathrm{MW} / \mathrm{m}^{2}$ as a function of total radiation fractions for an attached closed divertor. When the divertor spreading is of the order $4.5 \mathrm{~mm}$ or smaller, the total radiation fraction reaches a level, which seem very hard to maintain permanently. Therefore this suggests, that it is not possible to operate DEMO1 on a permanent basis under attached divertor conditions.

For detached divertor conditions measurements of the divertor heat flux profiles are more difficult and hence a similar empirical knowledge base has not yet been established. 
Table 2. Required divertor spreading $S_{\text {requ }}$ to allow for an outer peak heat flux density of $5 \mathrm{MW} / \mathrm{m}^{2}$ as a function of total radiation fractions for an attached closed divertor in DEMO1

\begin{tabular}{c|c|c|c|c|c}
$f_{\text {rad,tot }}$ & $\%$ & 85 & 90 & 95 & 97.5 \\
\hline$S_{\text {requ }}$ & $\mathrm{mm}$ & 20 & 14 & 7 & 3.2
\end{tabular}

Also modeling of detached divertor condition still lacks a predictive capability [19, 18].

\subsection{Loss of detachment}

Even if the detached divertor configuration is the default option there might be situations, in which the divertor is not detached. For instance such a situation could be caused by a failure of a component of the divertor control system like the impurity injection system. The code RACLETTE [20] is used to simulate such a situation as a sudden increase of the power flux density from $10 \mathrm{MW} / \mathrm{m}^{2}$ to a level $q_{1}$. RACLETTE evaluates the thermal response of all components involved in the heat removal process. It includes all key heat transfer process like evaporation, melting and radiation.

Figure 1a) shows the heat flux as a function of time and the critical heat flux at the interface between $\mathrm{Cu}$ and coolant after a sudden increase of the power flux density to $20 \mathrm{MW} / \mathrm{m}^{2}$ at $t=0 \mathrm{~s}$. The critical heat flux is defined as the maximum heat flux the system can remove for given pressure, velocity and inlet temperature of the coolant. Exceeding this critical heat flux value leads to boiling of the coolant at the pipe wall and to a sudden decrease of its cooling capability and to a sudden overheating of the component. Figure 1b) displays the evolution of the temperatures at the surface and at the interface between $\mathrm{Cu}$ and coolant for the same case.

Figure 1c) displays the time until the critical heat flux is reached as function of the enhanced power flux density for various levels of $\mathrm{W}$ thickness above the copper pipe. There is a certain lower threshold power flux density, below which there is infinite time until the critical heat flux is reached. This threshold decreases with $\mathrm{W}$ thickness above the $\mathrm{Cu}$ pipe. It increases by about $30 \%$ if the coolant velocity is increase from $12 \mathrm{~m} / \mathrm{s}$ (ITER value) to $20 \mathrm{~m} / \mathrm{s}$, which could be technical feasible. Figure 1c) shows that above the threshold, for instance for $5 \mathrm{~mm} W$ thickness the time to reach the critical heat flux drops below $1 \mathrm{~s}$ at about $30 \mathrm{MW} / \mathrm{m}^{2}$. In the case of $10 \mathrm{~mm} \mathrm{~W}$ thickness from about $35 \mathrm{MW} / \mathrm{m}^{2}$ surface melting poses the more restrictive limitation. Considering these time scales, it might be challenging to design a control system that is capable to detect such a failure and to react in a way that damages hampering further operation are avoided.

\subsection{Tungsten sputtering limit}

The $\mathrm{W}$ sputtering rate at the target plates is largely determined by the concentrations of impurities in the divertor plasma [21]. In order to assess this divertor limitation, an impurity concentration, which causes an appropriate level of core radiation, is evaluated. On the basis of this the necessary amount of an additional impurity to radiate in the 

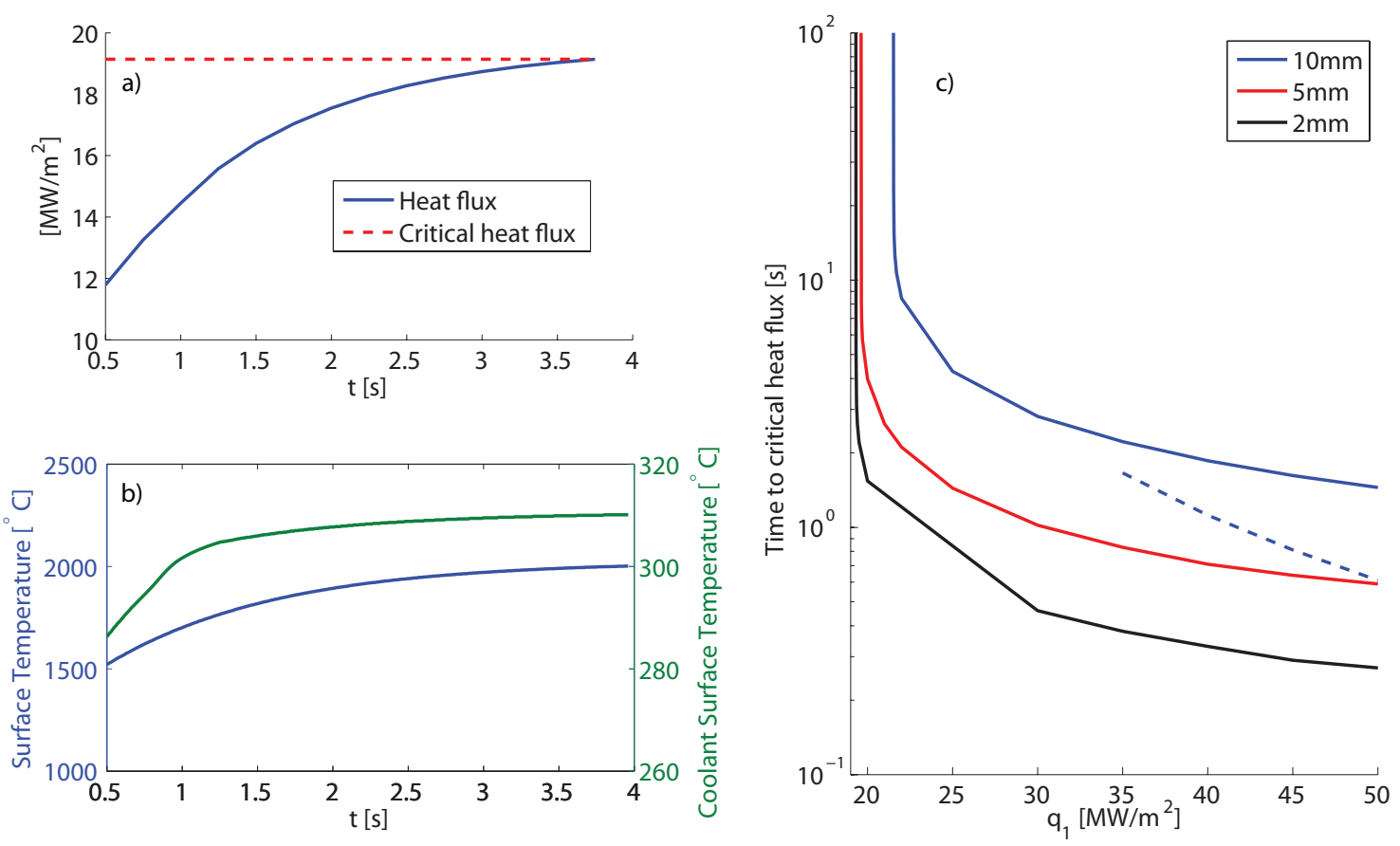

Figure 1. Results of RACLETTE calculations with mass flow rate $8 \times 10^{3} \mathrm{~kg} / \mathrm{m}^{2} / \mathrm{s}$, pressure $4 \mathrm{MPa}$, pipe inlet temperature $200^{\circ} \mathrm{C}$ and turbulence promotion factor 1.9 (swirl tube): a)Heat flux as a function of time and critical heat flux at the interface between $\mathrm{Cu}$ and the coolant after a sudden increase of the target power flux density to $20 \mathrm{MW} / \mathrm{m}^{2}$ at $t=0 \mathrm{~s}$ (W thickness: $5 \mathrm{~mm}$ ) .b) Evolution of temperatures at the surface and at the interface between $\mathrm{Cu}$ and coolant. c) Time until the heat flux reaches the critical heat flux as function of target power flux density and $\mathrm{W}$ thickness. The dashed line shows the surface melt limit for the cases, where it is more restrictive (W thickness: $10 \mathrm{~mm})$.

SOL and in the divertor is analyzed. Finally, the maximum temperature to satisfy at the same time the peak heat flux limit and the $\mathrm{W}$ sputtering limit is evaluated.

3.3.1. Core radiation The optimum value of core radiation is a balance between reducing the amount of power released to the SOL via ions and electrons and stable and controllable H-mode operation. Approaching the LH-threshold the confinement quality decreases significantly $[22,23]$. Considering results from Alcator C-Mod [23] it seems reasonable to request that the power over the separatrix $P_{\text {sep }}$ is about $120 \%$ of the LH-threshold power $P_{L H}$. This is close to what has been proposed in [24]. It remains to be analyzed, if this margin is sufficient to control the discharge stable in H-mode with the actuators and sensors available in DEMO.

In order to investigate the level of impurity seeding, which is necessary to fulfill the condition $P_{\text {sep }}=1.2 \times P_{L H}$ simulations with the ASTRA transport code [25, 26] have been carried out. As a theory based core transport model TGLF [27] has been employed, which results in observable stiff transport with a critical gradient in the 
order $R / L_{T} \approx 4-6$ following the basic gyro-Bohm scaling. TGLF has been validated on various machines [28, 29], however it is not clear, if it is appropriate for DEMO studies, in particular due to the high electron pressure $\left(\beta_{e} \approx 1 \%\right)$ and the relatively high fast alpha pressure $\left(p_{\alpha} / p_{t h} \approx 0.7\right)$. While ASTRA evolves the electron transport and calculates the D and T densities assuming quasi-neutrality, the coupled code STRAHL [30] evaluates the impurity transport. STRAHL assumes a finite impurity source just outside the separatrix. In the recent setup the neo-classical impurity transport is neglected. STRAHL evaluates for all impurities line radiation and Bremsstrahlung. The effect of a possible ELM activity has not been considered. A varying level of W impurity concentration between $10^{-5}$ and $10^{-4}$ has been used to simulate the influx of material sputtered from the plasma facing components. Additionally one or two seeding impurities have been used, where one of them has been scanned in concentration. The pedestal height has been set as described in section 2.2. Figure 2a) shows $P_{\text {sep }}$ versus $P_{\text {fus }}$ from these calculations and interpolations.

Figure 2b) shows electron density and temperature profiles for the calculation marked by the arrow in figure $2 \mathrm{a})\left(c_{W, \text { core }}=0.0011 \%, c_{A r, \text { core }}=0.92 \%\right)$. The W density profiles are peaked, which seems to be pessimistic when compared to a more detailed study for ITER [31] finding hollow W density profiles. Table 3 details the concentrations of the impurity combinations interpolated in a way that the condition $P_{\text {sep }}=1.2 \times P_{L H} \approx 180 \mathrm{MW}$ is fulfilled. The He concentration is close to $4 \%$. For all combinations but the last one an influx of the SOL seeding species Ne (section 3.3.2) into the core plasma has been ignored. For the last combination a relatively high divertor compression of 1:10 has been chosen (compare section 3.3.2). Also the power associated with line radiation and Bremsstrahlung is listed. The synchrotron radiation is in the order of 60MW. A lower level of $\mathrm{W}$ necessitates a higher level of seeding to reach the condition on $P_{\text {sep }}$. For the same level of $\mathrm{W}$ more Ar than $\mathrm{Kr}$ and more $\mathrm{Kr}$ than $\mathrm{Xe}$ is needed. This can be understood considering the fact that the temperature of maximum radiation increases with increasing charge number.

Next to the radiation capability the level of plasma dilution is another important aspect for the choice of seeding impurities. Figure 2a) shows that the reduction of power to the separatrix with increasing seeding is combined with a reduction of the fusion power $P_{\text {fus }}$ due to dilution. This effect significantly depends on the impurities species involved. For the three cases with $\mathrm{W}$ and Ar the fusion power is higher for higher $\mathrm{W}$ concentrations as this allows lower Ar concentrations and hence less dilution. At the low $\mathrm{W}$ level the fusion power is increasing when increasing the atomic number of the seed impurity $(\mathrm{Ar} \rightarrow \mathrm{Kr} \rightarrow \mathrm{Xe})$. The last impurity combination described in table 3 is the only case, in which the Ne influx towards inside the separatrix has been accounted for. Figure 2a) shows that adding even a small level of Ne as a second impurity species for SOL radiation causes a significant dilution.

3.3.2. Radiation from $S O L$ and divertor In the assessment of the radiation from the SOL and the divertor it is assumed that $30 \mathrm{MW}$ of power can be taken by the target. 
Table 3. Impurity concentrations on the magnetic axis to fulfill $P_{s e p}=1.2 \times P_{L H}$ and impurity concentrations in the SOL/divertor to fulfill $P_{\text {rad }, S O L}=1.2 \times P_{L H}-30 \mathrm{MW}=$ $150 \mathrm{MW}$ (section 3.3.2) and associated levels of core radiation (total impurity radiation and hydrogen Bremsstrahlung). Only the last combination accounts for Ne in the core.

\begin{tabular}{|c||c|c|c|c||c|c|c|}
\hline \multirow{2}{*}{$\begin{array}{c}\text { Core } \\
\text { radiators }\end{array}$} & \multicolumn{4}{c|}{ Concentrations } & \multicolumn{4}{c|}{ Core radiation } \\
\hline & $\begin{array}{c}\mathrm{W} \\
\text { Core }\end{array}$ & $\begin{array}{c}\text { Ar,Kr,Xe } \\
\text { Core }\end{array}$ & $\begin{array}{c}\text { Ar,Kr,Xe } \\
\text { SOL }\end{array}$ & $\begin{array}{c}\text { Ne } \\
\text { SOL }\end{array}$ & $\begin{array}{c}\text { Impurity radiation } \\
\text { W }\end{array}$ & $\begin{array}{c}\text { Brems- } \\
\text { strahlung }\end{array}$ \\
\hline & $\%$ & $\%$ & $\%$ & $\%$ & $\mathrm{MW}$ & $\mathrm{MW}$ & $\mathrm{MW}$ \\
\hline $\mathrm{W}+\mathrm{Ar}$ & 0.0011 & 0.92 & 2.76 & 4.00 & 12 & 139 & 25 \\
\hline $\mathrm{W}+\mathrm{Ar}$ & 0.0044 & 0.79 & 2.37 & 4.49 & 49 & 118 & 25 \\
\hline $\mathrm{W}+\mathrm{Ar}$ & 0.0089 & 0.57 & 1.71 & 5.29 & 102 & 85 & 26 \\
\hline $\mathrm{W}+\mathrm{Kr}$ & 0.0011 & 0.21 & 0.63 & 7.85 & 12 & 189 & 26 \\
\hline $\mathrm{W}+\mathrm{Xe}$ & 0.0011 & 0.08 & 0.24 & 9.02 & 13 & 212 & 27 \\
\hline $\mathrm{W}+\mathrm{Ar}+\mathrm{Ne}$ & 0.0009 & Ar:0.66, Ne:0.49 & 1.98 & 4.97 & 9 & Ar: $92, \mathrm{Ne:} 25$ & 22 \\
\hline
\end{tabular}
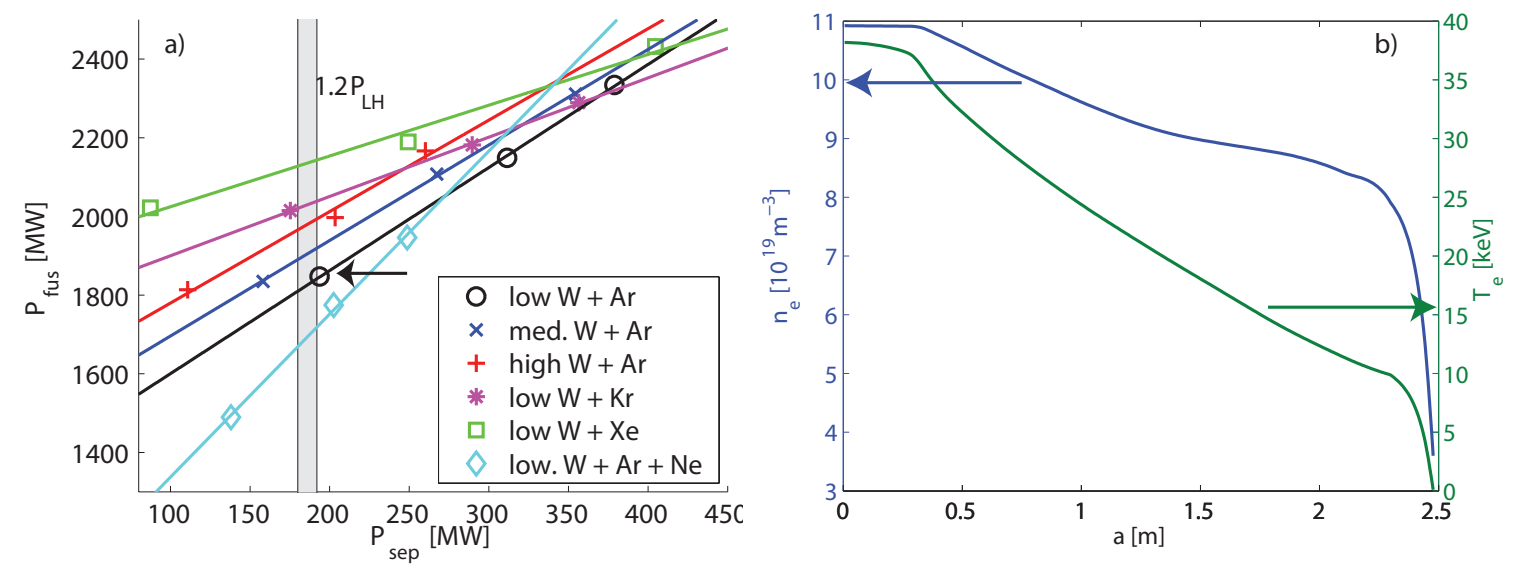

Figure 2. a) $P_{\text {sep }}$ versus $P_{\text {fus }}$ from calculations with ASTRA / TGLF / STRAHL for the impurity combinations listed in table 3 . Dots show simulation results and lines represent linear regressions. The vertical bar indicates the range of $1.2 \times P_{L H}$. b) Electron density and temperature profiles for the calculation marked by the horizontal arrow in a) $\left(c_{W, \text { core }}=0.0011 \%, c_{A r, \text { core }}=0.92 \%\right)$

This number is an estimation accounting for widening of the effective profile due to detachment and small ELMs. The remaining power of about $150 \mathrm{MW}$ needs to be radiated in the SOL and the divertor. This might be achieved via radiation of the impurity species chosen for main chamber radiation (e.g. Ar, Kr, Xe) alone or by adding a special SOL radiator (e.g. Ne). The divertor compression (i.e. the ratio between the impurity concentration in the main plasma and in the SOL and divertor plasma) depends on the divertor plasma condition, the pedestal plasma parameters and ELMs. In [32] based on experimental observations this ratio has been estimated as 1:3 with considerable uncertainties. We use this ratio as a first guess, for all impurity 
combinations but the last. Based on results from integrated divertor modeling it seems that there is a significant uncertainty on this ratio. This is especially the case for $\mathrm{Ne}$, which radiates mainly clearly above typical divertor temperatures.

The calculation of the radiation between midplane and divertor target is based on a 2-point model [33, 32]. The flux density of power radiated in the SOL may be calculated according to equation (5) in [32] as

$$
q_{\text {rad,SOL }}=n_{e, d} T_{e, \text { mid }}\left[2 \kappa_{0} Z_{e f f}^{-0.3} \int_{T_{e, t a r}}^{T_{e, \text { mid }}}\left(\sum_{i} c_{Z_{i}} L_{Z_{i}}\left(T_{e}, n_{e} \tau\right)\right) T_{e}^{0.5} d T_{e}\right]^{0.5}(5 .)
$$

For the separatrix density $n_{e, \text { mid }}=2.4 \times 10^{19} \mathrm{~m}^{-3}$ (one third of the pedestal top density) is used. For $T_{e, \text { mid }}$ a scaling on the basis of calculations with the integrated divertor modeling code B2-EIRENE [34] gives $200 \mathrm{eV}$, which is regarded as a relatively small and hence conservative value. $T_{e, t a r}=0 \mathrm{eV}$ has been assumed, which is close to the resulting temperatures based on the combined heat flux and $\mathrm{W}$ sputtering limitation (section 3.3.3). For the non-coronalness parameter $n_{e} \tau$ a value of $0.3 \times 10^{20} \mathrm{~m}^{-3} \mathrm{~ms}$ is used [32].

The total power, which is radiated in the SOL and the divertor can be calculated as

$$
P_{\text {rad }, S O L}=4 \pi(R+a) \lambda_{i n t} \frac{B_{\theta}}{B_{\phi}} q_{\text {max }, r a d},
$$

where $B_{\theta}$ and $B_{\phi}$ are the poloidal and toroidal components of the magnetic field at the outer midplane. For all the investigated impurity combinations the necessary concentration of $\mathrm{Ne}$ in the plasma edge is evaluated, in order to reach a total impurity radiation in SOL and divertor of $1.2 \times P_{L H}-30 M W$. While the assumption $T_{e, t a r}=0 \mathrm{eV}$ implies a slight overestimation of the power radiated in the SOL and the divertor, it is important to mention that the general approach neglects a number of relevant physics mechanisms like momentum loss processes. Combining all these considerations it is assumed that in total the approach overestimates the necessary Ne concentrations.

Table 3 details the SOL impurity concentrations for the impurity combinations regarded. The radiation by $\mathrm{W}$ and Xe from outside the separatrix has been regarded as negligible. Lower values of the core seeding concentration (i.e. higher values of the $\mathrm{W}$ concentration) and also changing the core seeding species from Ar to $\mathrm{Kr}$ and to $\mathrm{Xe}$ increases the required amount of Ne to be seeded in the SOL.

3.3.3. Combined divertor peak heat flux / $W$ sputtering limit On the basis of the concentrations listed in table 3 the $\mathrm{W}$ sputtering yield has been calculated. For this the Bohdansky formula [35] has been used:

$$
\begin{aligned}
& Y\left(E_{0}, \alpha=0^{\circ}\right)=Q \times S_{n}(\epsilon)\left[1-\left(\frac{E_{t h}}{E_{0}}\right)^{2 / 3}\right]\left(1-\frac{E_{t h}}{E_{0}}\right)^{2} \\
& S_{n}(\epsilon)=\frac{0.5 \ln (1+1.2288 \epsilon)}{\epsilon+0.1728 \sqrt{\epsilon}+0.008 \epsilon^{0.1504}} \\
& \epsilon=E_{0} \frac{M_{2}}{M_{1}+M_{2}} \frac{a_{L}}{Z_{1} Z_{2} e^{2}}=E_{0} / E_{T F}
\end{aligned}
$$


Table 4. Coefficients used for the sputtering yield calculation

\begin{tabular}{|c||c|c|c|}
\hline Species & $\begin{array}{c}E_{T F} \\
\mathrm{eV}\end{array}$ & $\begin{array}{c}E_{t h} \\
\mathrm{eV}\end{array}$ & $Q$ \\
\hline $\mathrm{D}$ & 9925 & 201 & 0.0345 \\
$\mathrm{Ne}$ & 119127 & 33.49 & 2.16 \\
$\mathrm{Ar}$ & 246680 & 33.44 & 5.32 \\
$\mathrm{Kr}$ & 636771 & 42.58 & 13.77 \\
\hline
\end{tabular}
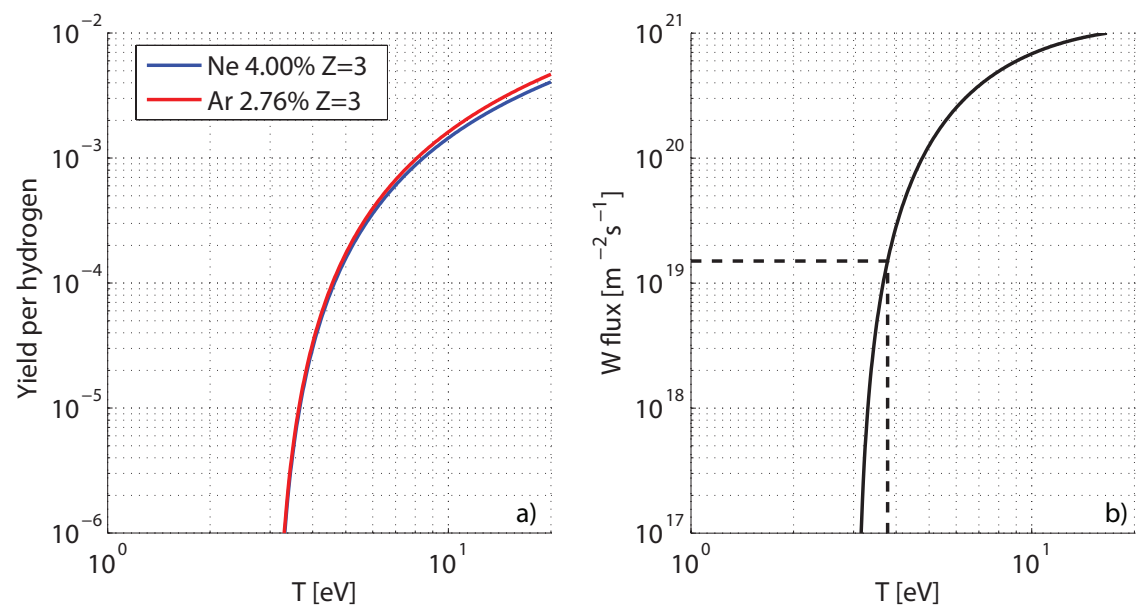

Figure 3. a) W sputtering yield per hydrogen as a function of temperature for the impurity combination $c_{A r, S O L}=2.76 \%$ (at charge state $\mathrm{Z}=3$ ), $c_{N e, S O L}=4.00 \%$ (at charge state $\mathrm{Z}=3$ ). b) Total $\mathrm{W}$ sputtering flux for the same impurity mix as a function of temperature. The flux of impacting hydrogen has been calculated based on a heat flux of $5 \mathrm{MW} / \mathrm{m}^{2}$ and a sheath transmission coefficient of 8 .

$$
a_{L}=0.4648\left(Z_{1}^{2 / 3}+Z_{2}^{2 / 3}\right)^{-1 / 2} \AA .
$$

Here $Y$ is the sputtering yield, $E_{0}$ is the projectile energy, $E_{t h}$ is the threshold energy, $\epsilon$ is the reduced energy, $S_{n}$ is the nuclear stopping cross section, $Q$ is a fit parameter and $a_{L}$ is the screening length for the interaction potential. The subscripts in equations 9 and 10 denote projectile (1) and target (2). The employed coefficients are shown in table 4. A charge state of $Z=3$ is used for Ne, Ar and Kr. Figure 3a) shows the results for the first combination in table $3\left(c_{A r, S O L}=2.76 \%, c_{N e, S O L}=4.00 \%\right)$. For the conversion into the $\mathrm{W}$ sputtering flux, information on the hydrogen flux to the divertor is derived as in [32]. In addition to a peak heat flux limit of $5 \mathrm{MW} / \mathrm{m}^{2}$, additionally it is assumed that $2 \mathrm{MW} / \mathrm{m}^{2}$ of this power flux density is covered already by loads due to radiation. The power deposited by neutrals [37] and neutrons has not been taken into account. Furthermore, the calculation has been based on a sheath transmission factor of 8 . The product of the yield per hydrogen and the hydrogen flux, which are both temperature dependent, gives the $\mathrm{W}$ sputtering flux. Figure $3 \mathrm{~b}$ ) shows the total $\mathrm{W}$ sputtering flux for the impurity mix used in figure 3a). Further investigations show that the difference due to the assumption of full molecular recombination is negligible.

A value for the maximum acceptable $W$ sputtering flux is obtained on the basis of 
several assumptions. For the maximum thickness of the W layer that may be eroded from the target $3 \mathrm{~mm}$ is assumed. A replacement of the divertor cassettes due to deterioration of material properties by neutron irradiation is considered to be necessary after 2 full power years. For the total redeposition rate a pessimistic value of $80 \%$ [38] has been assumed. The resulting limit for the $\mathrm{W}$ sputtering flux is $1.5 \times 10^{19} \mathrm{~m}^{-2} \mathrm{~s}^{-1}$. For the impurity combinations listed in table 3 with $\mathrm{Ar}$ or $\mathrm{Kr}$ as core seeding impurity the combined heat flux density / W sputtering limitation necessitates divertor electron temperatures not higher than than $3.9 \mathrm{eV}$.

\section{Divertor limitations during ELMs}

ELMs are associated with high doses of energy and particles released from the plasma within times, which are shorter than $1 \mathrm{~ms}$. It is the objective to incorporate a method to suppress or mitigate ELMs into the DEMO design. Despite several of these methods being under development, none of them has reached a level of maturity to be sure that ELM control or mitigation in DEMO will be possible. Furthermore even if a reliable method would be developed, there might be always the possibility of having a low number of natural ELMs for instance due to operational failures.

It has been shown in ASDEX Upgrade that the ELM type is largely determined by the normalized collisionality $\nu^{*}[39,40]$. Here the volume averaged value of $\nu^{*}$ has been used, which is similar to the local value for $\rho_{\text {pol }} \approx 0.7-0.8$. While for $\nu^{*}>1$ type III ELMs have been dominant, for $\nu^{*}<0.3$ type I ELMs have been dominant. In the transport calculations described in section 3.3.1 $\nu_{\text {ped }}^{*}$ is lower than 1 for all impurity concentrations. Hence without a working mitigation method for DEMO type I ELMs are to be expected. This is also supported by the consideration that the pedestal collisionality is very low and thus the edge bootstrap current is large. These are conditions, in which the peeling-ballooning instability is expected, which is deemed to cause type I ELMs.

The energy loss per type I ELM is an important quantity to extrapolate to DEMO. Experiments at JET suggest that at values of $\nu^{*}$ around 0.1 the ELM loss energy is about $20 \%$ of the pedestal stored energy [41]. It is not clear that this can also be applied for DEMO. However the paradigm of stiff gradients would support such an assumption. Application to DEMO leads to a value for uncontrolled ELMs in the order of 100MJ. This is more than 100 times above the maximum ELM size observed in JET.

Besides other divertor limitations due to ELMs, which are not discussed in this publication, the energy density limit takes a central part. A value of $0.5 \mathrm{MJ} / \mathrm{m}^{2}$ is used to estimate the tolerable ELM energy limit for DEMO, which is identical to the value used for ITER. However this value could be further reduced, as W cracking by longterm cycling due to ELMs can lead to a strong deterioration of the surface, which could lead to a lower energy density limit. The ELM wetted area is estimated based on the inter-ELM wetted area of $1.4 \mathrm{~m}^{2}$ (section 3.1). The broadening of the wetted area during the ELM with respect to the inter-ELM level has been observed in JET to depend on 


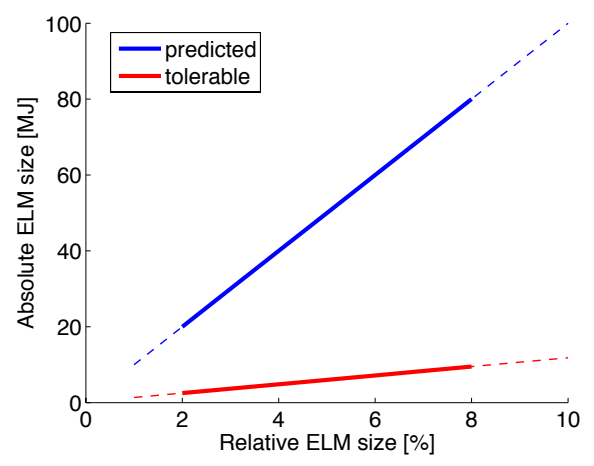

Figure 4. Predicted ELM size and tolerable ELM size as a function of ELM size relative to the total stored energy for DEMO1: The total stored energy has been set to 1GJ. The solid lines illustrate the range in relative ELM size, for which data on broadening at JET has been collected [42].

the ELM size relative to the total stored energy [42]. It is now assumed that a similar correlation is valid also for DEMO. Figure 4 shows that for DEMO1 the absolute ELM size exceeds the tolerable ELM size by a nearly constant factor of about 8 . Even if the ELM size is reduced by a certain factor, the tolerable ELM size might be reduced by about the same factor. This is the case because it is assumed that the ELM wetted area scales linearly with ELM energy loss. If the broadening would have a bit weaker scaling, the ratio of predicted and tolerated ELM size would depend on the relative ELM size. Also it has to be noted that due to the size of DEMO and the expected profile peaking a normalization by the total stored energy as applied in [42] might lead to a pessimistic prediction for DEMO.

The high ratio of predicted and tolerable ELM size raises the question, if the concept of reducing the ELM size below a certain threshold without changing the ELM type is appropriate for DEMO. It is also conceivable that only a method is effective, that leads to very small but high frequent plasma energy releases. Despite some doubts, it needs to be investigated, if scenarios with very small or no ELMs such as the QH mode [43] or type II ELM [44] regimes are an option for DEMO. Furthermore the effectiveness of RMP ELM mitigation in ITER and in DEMO is of central interest.

\section{Discussion}

Operation of DEMO with a conventional W divertor has to respect several limitations. A subset of these has been discussed in this paper. The extrapolation of these limitations to DEMO is challenged by a number of significant uncertainties, that often relativize quantitative statements. These uncertainties need to be the origin of future work. It has been tried to make conservative assumptions in all areas of uncertainty. This leads to following main conclusions:

- Attached divertor operation at full fusion power and a total radiative power fraction of $90 \%$ or lower would lead to divertor damage if sustained for longer than a few 
seconds.

- The combined divertor $\mathrm{W}$ erosion / heat flux limit results in the need for divertor electron temperatures of less than $4 \mathrm{eV}$.

- If no effective ELM control method is incorporated, type I ELMs need to be expected. Their size is estimated to be about one order of magnitude above the tolerable ELM size for a large range of relative ELM sizes. This means that DEMO operation is is not possible without effective ELM control method.

The main conclusion to draw in terms of inter-ELM limitations is that the default divertor operation scenario should be detached or semi-detached. However as pointed out before integrated modeling of main plasma, SOL and divertor has not yet reached the capability to predict the behavior of a detached or semi-detached divertor. Once obtaining this capability, it is particular desirable to analyze the question, if divertor electron temperatures in the range of a few $\mathrm{eV}$ are achievable in DEMO. Also the empirical knowledge of the peak power flux density with a detached or semi-detached divertor is much less developed as for attached divertor conditions and needs to be extended. For the development of the DEMO design parameters reasonable boundary conditions to account for the divertor limitations need to be included in system codes now. In lack of the capability to predict the behavior of detached divertor it has been proposed to use $P_{\text {sep }} / R$ as a quantity that describes the level of the divertor challenge [18]. Values of up to $7 \mathrm{MW} / \mathrm{m}$ have been demonstrated in ASDEX Upgrade. ITER is expected to have a value of $14 \mathrm{MW} / \mathrm{m}$ and for DEMO we suggest to use $17 \mathrm{MW} / \mathrm{m}$ as a boundary condition to allow for divertor operation. Hence it is of high importance to further extend the range in $P_{\text {sep }} / R$ in existing devices with realistic divertor geometry.

A number of significant limitations on the operation with a conventional divertor in DEMO is discussed in this publication. In order to consolidate these results and to understand, if these limitations can be respected simultaneously a concentrated activity in the area of modeling and also dedicated experiments have to be carried out. Due to the current lack of predictive capability it is also necessary to investigate alternative divertor concepts in parallel.

\section{References}

[1] Romanelli $\mathrm{F}$ et al Fusion Electricity - A roadmap to the realisation of fusion energy ISBN 9783-00-040720-8

[2] Hirai T et al 2013 Fusion Engineering and Design 881798 - 1801

[3] Greenwald M 2002 Plasma Physics and Controlled Fusion 44 R27

[4] Federici G et al Fusion Engineering and Design - accepted

[5] Lang P et al 2012 Nuclear Fusion 52023017

[6] Saibene G et al 2002 Plas.Phys.Contr.Fusion 44 1769-1799

[7] Huber A et al 2013 Journal of Nuclear Materials 438, Supplement S139 - S147

[8] Bernert M et al 2013 40th EPS Conference on Controlled Fusion and Plasma Physics 40

[9] Schneider P et al 2013 Nuclear Fusion 53073039

[10] Eich T et al 2011 Phys. Rev. Lett. 107215001

[11] Herrmann A and Gruber O 2003 Fusion Science and Technology 44 569-577 
[12] Makowski M A et al 2012 Physics of Plasmas 19056122

[13] Eich T et al 2013 Nuclear Fusion 53093031

[14] Eich T et al 2013 Journal of Nuclear Materials 438 S72 - S77

[15] Sieglin B et al 2013 Plasma Physics and Controlled Fusion 55124039

[16] Lowry C PhD in Physics, 1999

[17] Eich T et al 2007 Plasma Physics Contr.Fusion 49 p.573-604

[18] Kallenbach A et al 2012 Nuclear Fusion 52122003

[19] Wischmeier M et al 2011 Journal of Nuclear Materials 415 S523 - S529 Proceedings of the 19th International Conference on Plasma-Surface Interactions in Controlled Fusion

[20] Raffray A and Federici G 1997 Journal of Nuclear Materials 24485 - 100

[21] Kallenbach A et al 2011 Journal of Nuclear Materials 415 S19 - S26 Proceedings of the 19th International Conference on Plasma-Surface Interactions in Controlled Fusion

[22] Loarte A et al 2011 Physics of Plasmas (1994-present) 18056105

[23] Hughes J et al 2011 Nuclear Fusion 51083007

[24] Zohm H et al 2013 Nuclear Fusion 53073019

[25] Pereverzev G V et al 1991 Lab Report, Max-Planck-Institut fuer Plasmaphysik 5/42

[26] Fable E et al 2013 Plasma Physics and Controlled Fusion 55124028

[27] Staebler G M, Kinsey J E, and Waltz R E 2007 Physics of Plasmas (1994-present) 14055909

[28] Kinsey J E, Staebler G M, and Waltz R E 2008 Physics of Plasmas (1994-present) 15055908

[29] Sommer F et al 2012 Nuclear Fusion 52114018

[30] Dux R 2006 Lab Report, Max-Planck-Institut fuer Plasmaphysik 10/30

[31] Dux R et al 2013 40th EPS Conference on Controlled Fusion and Plasma Physics 40

[32] Kallenbach A et al 2013 Plasma Physics and Controlled Fusion 55124041

[33] Post D, Abdallah J, Clark R E H, and Putvinskaya N 1995 Physics of Plasmas 2 2328-2336

[34] Pacher H, Kukushkin A, Pacher G, Kotov V, and Reiter D 2011 Journal of Nuclear Materials 415 S492 - S496

[35] Bohdansky J 1984 Nuclear Instruments and Methods in Physics Research Section B: Beam Interactions with Materials and Atoms 2587 - 591

[36] Eckstein W et al 1982 IPP-Report $\mathbf{9 / 8 2}$

[37] Hoshino K et al 2012 Contributions to Plasma Physics 52 550-554

[38] Dux R et al 2009 Journal of Nuclear Materials 390391858 - 863

[39] Suttrop W et al 2000 Plas.Phys.Contr.Fusion 42 A1-A14

[40] Ryter F et al 2008 Journal of Physics: Conference Series 123012035

[41] Loarte A et al 2003 Plasma Physics and Controlled Fusion 45 1549-1569

[42] Thomsen H et al 2011 Nuclear Fusion 51123001

[43] Burrell K H et al 2002 Plasma Physics and Controlled Fusion 44 A253

[44] Doyle E J et al 1991 Physics of Fluids B: Plasma Physics 3 2300-2307 